\title{
Double-chambered right ventricle: a commonly overlooked diagnosis
}

\author{
Joe KT Lee *, KL Tsui
}

Hong Kong Med J 2015;21:84.e1-2

DOI: 10.12809/hkmj134187

A 72-year-old woman presented with decreased exercise tolerance since 2007. Based on a transthoracic echocardiogram (TTE) in another hospital, the patient was diagnosed to have perimembranous ventricular

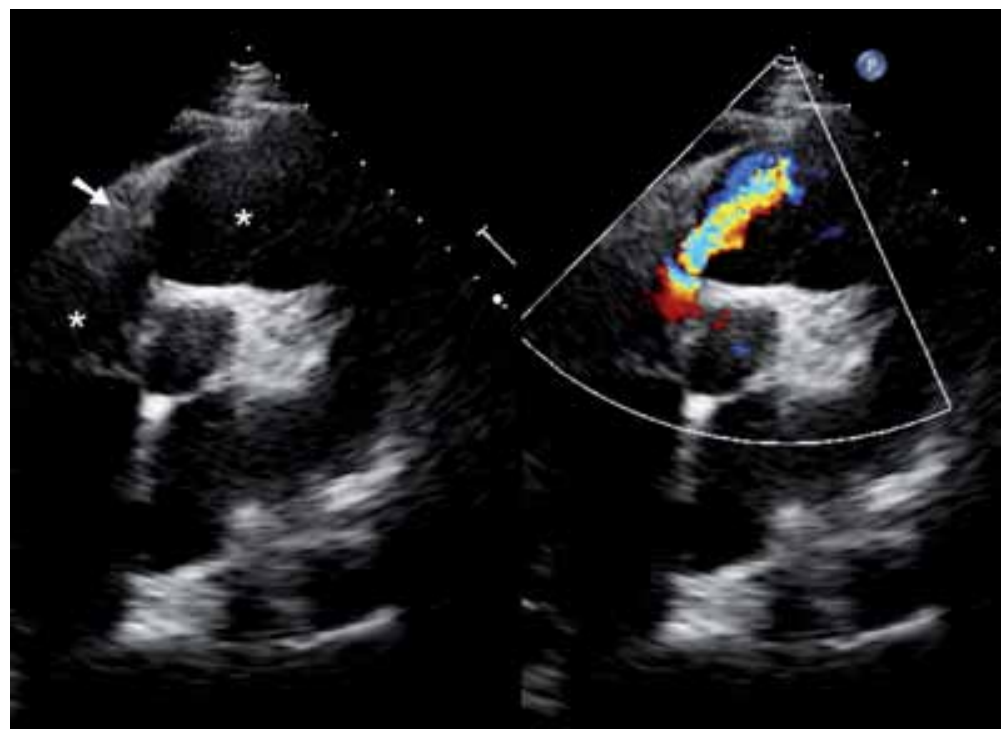

FIG I. The mid-cavitary stenosis (arrow) divides the right ventricle into two separate chambers (asterisks), with the systolic turbulent jet across the stenosis (parasternal short axis view of transthoracic echocardiogram)

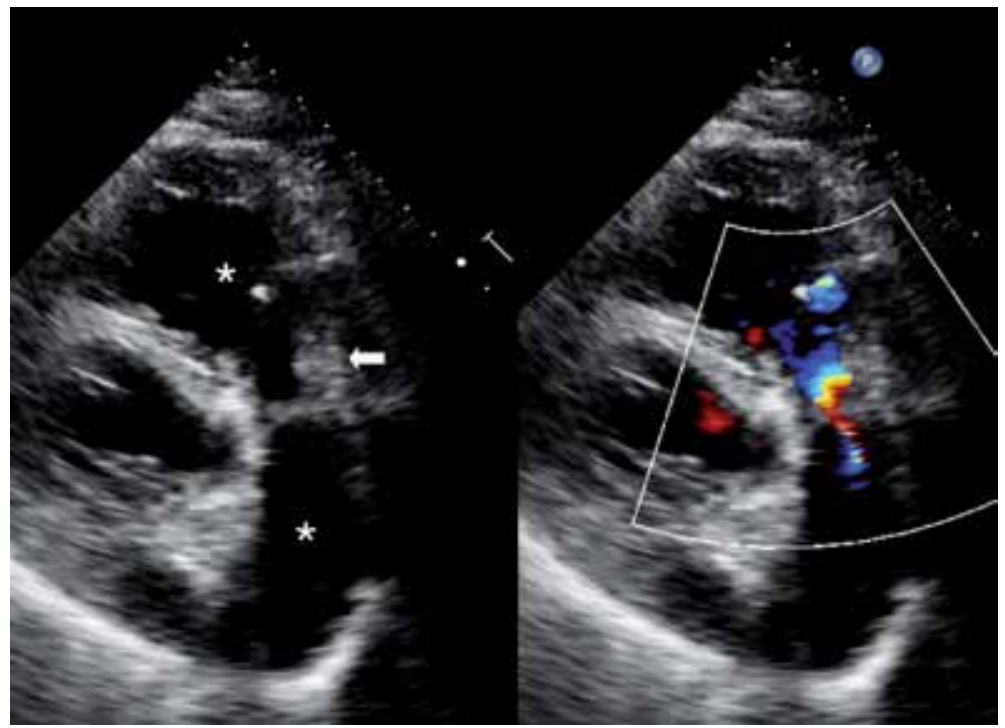

FIG 2. On subcostal short axis view of transthoracic echocardiogram, a more parallel alignment of the turbulent jet to the transducer can be obtained, which allows more accurate pressure gradient assessment by Doppler between the two right ventricular chambers (asterisks) across the stenotic band (arrow) septal defect (VSD) with pulmonary hypertension. Upon referral to our unit in 2013, a more meticulous TTE examination revealed a mid-cavitary stenosis in the right ventricle (RV) which was best appreciated in the parasternal short axis and the subcostal short axis view (Figs 1 and 2 ). The systolic pressure gradient measured by continuous-wave Doppler between the two RV chambers was markedly elevated to $80 \mathrm{~mm}$ $\mathrm{Hg}$. There was also severe tricuspid regurgitation with a dilated RV. The findings were confirmed on a transoesophageal echocardiogram (TEE) and the diagnosis was revised as double-chambered right ventricle (DCRV) with perimembranous VSD (Fig 3). A right heart catheterization study showed a stenotic band over the right ventricular outflow tract (RVOT) [Fig 4]. The systolic pressure at the proximal $\mathrm{RV}$ chamber was markedly elevated to $75 \mathrm{~mm} \mathrm{Hg}$. However, we failed to manipulate the catheter across the stenotic band to measure the pressure gradient across the two chambers. The coronary angiographic results were normal. The patient was referred to cardiothoracic surgeons and open heart surgery was undertaken. After right ventriculotomy, the anomalous infundibular muscle bundle and a small perimembranous VSD were identified. The obstructive muscle bundle was resected and VSD was repaired. The tricuspid valve was repaired by means of annuloplasty. In postoperative TTE, the previously noted high pressure gradient across the RVOT was no longer present. The right ventricular systolic pressure normalised to $15 \mathrm{~mm} \mathrm{Hg}$.

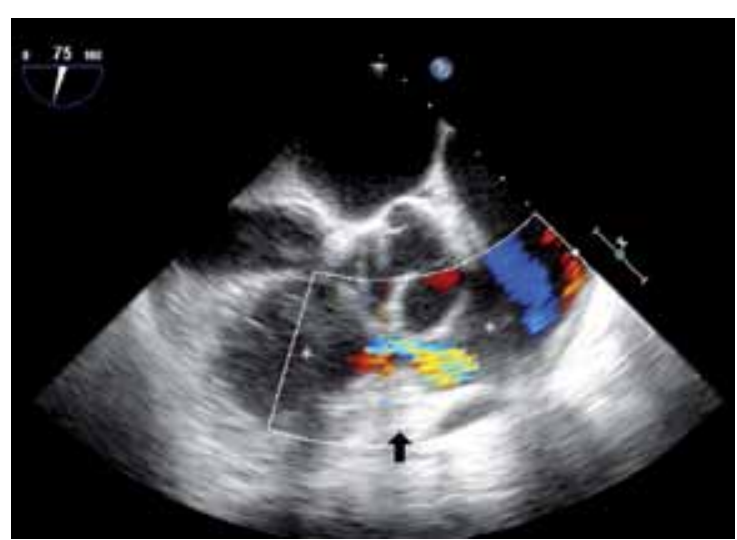

FIG 3. The systolic turbulent jet across the mid-cavitary stenosis (arrow) in between the two right ventricle chambers (asterisks) $\left[75^{\circ}\right.$ on transoesophageal echocardiogram] 


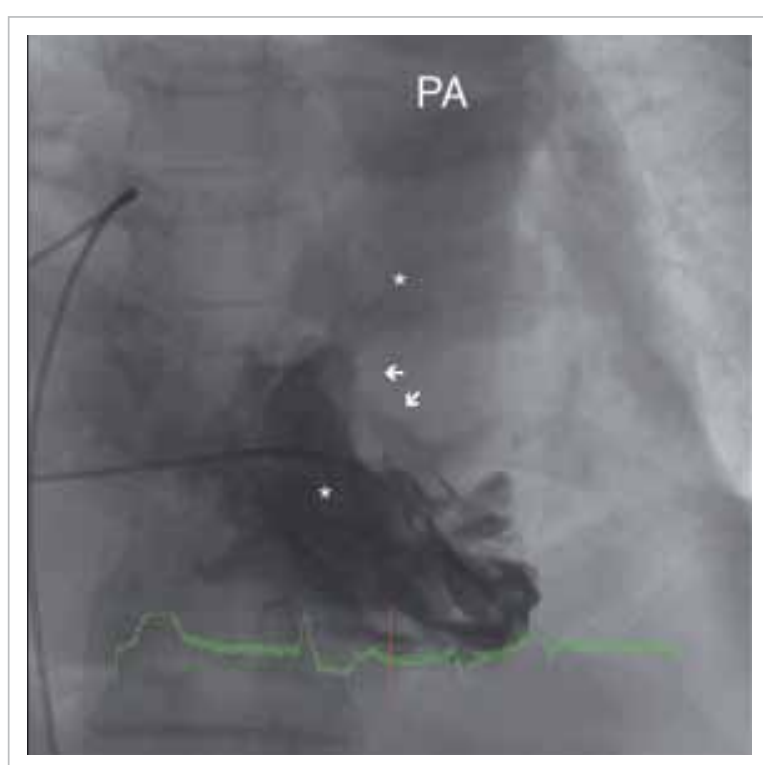

FIG 4. The right-heart ventriculogram on postero-anterior projection reveals a stenotic band in the right ventricular outflow tract (arrows) and the two right ventricle chambers (asterisks)

Abbreviation: PA = main pulmonary artery

\section{Discussion}

Double-chambered RV is characterised by the presence of an anomalous muscle bundle (AMB), which divides the RV into two separate chambers, namely, the proximal high-pressure and the distal low-pressure chambers. The AMB is considered a hypertrophied moderator band or the accentuated septoparietal trabeculation., ${ }^{1,2}$ These congenital anatomical substrates and other acquired haemodynamic factors lead to the development of DCRV.

Double-chambered RV is an uncommon condition and it is only seen in $0.5 \%$ to $2 \%$ of all cases of congenital heart disease. ${ }^{3}$ Most cases are diagnosed in childhood or adolescence before the age of 20 years. The occurrence in adults is rare and has only been described in case reports and small case series. About $80 \%$ to $90 \%$ of DCRV cases are associated with VSD, or sometimes with other congenital cardiac anomalies. Patients with DCRV usually present with shortness of breath and decreased exercise tolerance, but they may also have atypical symptoms such as chest pain, dizziness, and syncope.
The irregular shape and retrosternal position of $\mathrm{RV}$, and its close proximity to the precordium impose diagnostic difficulty by TTE, especially in adults. Quite often, the mid-cavitary turbulent jet on TTE is mistaken as an intracardiac shunt, and the high RV systolic pressure is falsely interpreted as pulmonary hypertension. In a case series, only $15.6 \%$ of patients with DCRV could be correctly diagnosed by TTE. ${ }^{3}$ A high clinical suspicion and awareness of this clinical entity are required for precise diagnosis. The subcostal view of TTE may sometimes provide better visualisation of the RV and RVOT. It also allows better alignment of the turbulent jet for pressure gradient measurement. When TTE is not confirmative, TEE and cardiac catheterization serve as complementary tools. The use of cardiac magnetic resonance imaging as non-invasive assessment is now emerging as an alternative diagnostic modality for DCRV.

Surgical repair of RV by resection of the AMB is indicated in symptomatic patients, and it yields excellent long-term haemodynamic and functional results. ${ }^{4}$ Surgical treatment is also suggested in asymptomatic patients who have significantly elevated midventricular pressure gradient, that is, $>40 \mathrm{~mm} \mathrm{Hg}$ as the obstruction can progress rapidly over just a few years. ${ }^{5}$

JKT Lee *, MRCP (UK), FHKAM (Medicine)

KL Tsui, FRCP (Edin, Glasg), FHKAM (Medicine)

Department of Medicine, Pamela Youde Nethersole Eastern Hospital, Chai Wan, Hong Kong

* Corresponding author: jktlee@gmail.com

\section{References}

1. Wong PC, Sanders SP, Jonas RA, et al. Pulmonary valve-moderator band distance and association with development of double-chambered right ventricle. Am J Cardiol 1991;68:1681-6.

2. Alva C, Ho SY, Lincoln CR, et al. The nature of the obstructive muscular bundles in double-chambered right ventricle. J Thorac Cardiovasc Surg 1999;117:1180-9.

3. Hoffman P, Wojcik AW, Rozanski J, et al. The role of echocardiography in diagnosing double chambered right ventricle in adults. Heart 2004;90:789-93.

4. Telagh R, Alexi-Meskishvili V, Hetzer R, et al. Initial clinical manifestations and mid- and long-term results after surgical repair of double-chambered right ventricle in children and adults. Cardiol Young 2008;18:268-74.

5. Oliver JM, Garrido A, Gonzalez A, et al. Rapid progression of midventricular obstruction in adults with doublechambered right ventricle. J Thorac Cardiovasc Surg 2003;126:711-7. 